\title{
Primary Anorexia Nervosa or Weight Phobia in the Male: Report on 13 Cases
}

\author{
A. H. CRISP, D. A. TOMS
}

possibly significant homosexual conflict preceding the onset of the disorder in up to $50 \%$ of their male patients (Crisp, 1967;

\section{Summary}

The cases of 13 men with anorexia nervosa are reported. While the disorder as seen in the clinic is much less common in males than females this may not be a true reflection of the differential sex or overall prevalence rates. The disorder is found to have the same basic characteristics in the male as in the female: namely, a phobic avoidance of normal weight associated with elective carbohydrate starvation and emaciation. As in the female the mechanism appears to develop out of normal adolescent dieting behaviour and to arise as a means of avoiding overwhelming psychosocial maturational demands of adolescence. Premorbid and family features include a state of overnutrition and a high degree of family psychopathology reflected in high rates of parental psychiatric morbidity and marital difficulty.

\section{Introduction}

Primary anorexia nervosa in the female is thought to be rare. This conclusion is probably greatly affected by a central aspect of the disorder-the subject's reluctance or intense fear of change and her insistence that she is not ill. Many therefore avoid becoming patients, even sometimes to the point of death, and others who are precipitated into medical care successfully conceal the true basis of their symptomatology and behaviour. Nevertheless, patients from the background with the greatest prevalence of the disorder-namely, adolescent girls from social classes I and II-will sometimes convincingly acknowledge that at any one time between one in 50 and one in 100 of their fellow schoolgirls display a similar severe and seemingly indisputable form of the disorder. The disorder may be becoming more common, but this is hard to establish.

Series of 50 or more women have now been carefully documented and described by several clinical research workers. All agree that the disorder is much more rare in the male (Crisp, 1967; Dally, 1969; Theander, 1970), reports of the sex ratio ranging from 1 in 10 to 1 in 20. Bliss and Branch (1960) suggested that the male prevalence rate may be greater than this but that it is masked by a greater reluctance on the part of clinicians to diagnose the condition in young men. In particular, secondary amenorrhoea cannot draw attention to the need for further inquiry into the psychological, social, and nutritional history of the individual. Dally (1969) reviewed some of the scanty literature on male anorexia nervosa and described six more cases. Bruch (1971) added another five cases, which she carefully distinguished from four other male patients with anorexia and cachexia associated with other psychiatric illnesses and without the central characteristics of primary anorexia nervosa. These she identified as refusal to eat predominantly carbohydrate foods in the pathological pursuit of thinness together with major body-image and somatic-perceptual disturbances.

Several authors have drawn attention to the presence of

St. George's Hospital Medical School, St. George's Hospital, London S.W.17

A. H. CRISP, M.D., M.R.C.P., Professor of Psychiatry

D. A. TOMS, M.R.C.P., D.P.M., Senior Registrar in Psychiatry drug and alcohol dependence and "compulsive" overeating have been emphasized in several reported case histories. Dally (1969) concluded that the disorder carried a worse prognosis in the male. In all about 50 cases have been described in the literature over the past two centuries in more or less detail.

This report concerns the cases of 11 male patients studied in detail over the past 10 years together with two other patients included after attention had been drawn to their case histories because of their family association with women with primary anorexia nervosa receiving treatment under our care. All the subjects were considered to have severe and definite primary anorexia nervosa-namely, a state of emaciation associated with inability to ingest or retain ingested food and characteristically accompanied by low basal metabolism, daytime and nocturnal restlessness, and preoccupation with food. Associated with the inability to eat was a resistance to eating fattening foods, often arising from an established fear of fatness, but always linked with a progressively single-minded pursuit of thinness associated with a developing fear of and determination to avoid normal adolescent and adult weight. This fear and the consequent feeding disorder had usually been concealed or denied at first.

Seven of the patients received a period of inpatient care during which their weight was restored to the level of their matched population mean weight, while at the same time they and their families were given psychotherapy. The four other patients were seen and treated as outpatients. Details of the clinical presentation and course of some of these cases and the other two subjects are given elsewhere (Crisp and Roberts, 1962; Crisp, 1967; Toms and Crisp, 1972). The data presented here have to do with some of the factors previously found to be important in female anorexia nervosa (Crisp, 1970). They comprise (a) some social, family, and clinical characteristics of the total group, and $(b)$ results of the investigation during treatment of one patient whose distorted growth we were able to examine in some detail.

\section{Method}

Information about psychological and social aspects of patients and especially about their personal histories is notoriously inaccurate. This report reflects part of a larger investigation into such aspects in female patients with primary anorexia nervosa, and throughout we have tried to improve the quality of the data by using as many sources as possible. This information was derived from the patients and their families, usually over a prolonged period, and concerns the background of the subjects and their families, their clinical state, and the course of the disorder. In some instances the subjects have been compared with subgroups or the total group in 160 cases in female patients seen and documented (Crisp, 1970) by A. H. C. over the same period.

\section{Family Background}

SOCIAL CLASS

The parental social class in Case 12 was not recorded. Six of the other 12 subjects came from a social class I or II back- 
ground and the other six from a social class III, IV, or V background (father's occupation, Registrar General's classification). This can be compared with the even greater predominance of social class I and II backgrounds ( 40 among the first 55 subjects) in the parallel series of female patients, but this difference does not reach the $5 \%$ level of significance.

\section{BIRTH ORDER}

The reported birth order in 12 of the subjects is shown in Table I. Seven were first-born and five were second-born. When this finding is compared with the reported birth order of the first 55 subjects in the female series (22 first-born, 22 second-born, 7 born third or later, and 4 unknown) the tendency for the males to be more often first-born is seen not to reach statistical significance.

TABLE I-Birth Order in 13 Male Subjects with Primary Anorexia Nervosa or Weight Phobia (Reported Live Births)

\begin{tabular}{c|ccc}
\hline Case No. & \multicolumn{3}{|c}{ Birth Order } \\
\hline 1 & Brother & S & Sister \\
2 & 1 & Sister & \\
3 & Sister & \\
4 & Sister & 2 & \\
5 & 1 & Sister & Sister \\
6 & 1 & Brother & \\
7 & 1 & 2 & \\
8 & Sister & Brother \\
9 & 1 & Brother* & Sister* \\
10 & 1 & Brother & $?$ \\
11 & 1 & Sister** & Brother \\
12 & & &
\end{tabular}

- Sibling with anorexia nervosa.

\section{PARENTAL AGE}

The mean paternal age was 31 years at the time of the probands' birth and the mean maternal age 29 years. The standard deviations were small.

\section{FAMILY NUTRITIONAL HISTORY}

The weight and shape of most of the parents was not abnormal. Several parents were intensely preoccupied with their physical fitness and with maintaining normal weight, and in association with this several mothers and fathers severely restricted their carbohydrate intake either consistently or sporadically. Of three fathers working with food, however, two were chefs and chronically obese.

The occurrence of primary anorexia nervosa among other members of the family was remarkable. From Table $I$ it is seen that three siblings had had the condition. In Cases 11 and 13 the disorder was in the sister and was carefully documented, in one instance by ourselves. In Case 10 the disorder had almost certainly occurred in the second-born brother. In these three instances the disorder developed in sequence between the siblings concerned. In Case 10 first the eldest brother (under our care) developed the condition. As he recovered his brother developed the disorder. In Case 11 it is likely that the elder brother (under our care when he reached the age of 26 years) had developed the disorder first. During what proved to be a remission in his condition his younger sister developed the disorder, which lasted about three years. As she recovered he redeveloped the disorder in a severe form, which persisted. In Case 13 the occurrence of the disorder in the elder brother was brought to our attention by the presentation with it of his sister. She had been ill for about three years after his recovery.

Finally, Case 12 is included in this series mainly because we think the facts of this case provide evidence for the importance of environmental background factors in the disorder. He was a very thin 46-year-old man who presented as the adoptive father of a 14-year-old patient with primary anorexia nervosa. He was himself financially successful as a sweet-food manufacturer. It was evident that he had been the subject of primary anorexia nervosa for many years. His marriage was stable but infertile and possibly unconsummated, and it had resulted in adoption of a baby who was by now the adolescent patient presenting with the disorder. A second girl with primary anorexia nervosa subsequently presented to A.H.C., and one of the features in her history was a period in early adolescence when she had stayed with this family as an evacuee during the second world war. This period had ended about five years before the onset of her anorexia nervosa; she had become progressively obese at that time.

\section{PSYCHIATRIC HISTORY IN THE FAMILY}

There was a broad but not necessarily remarkable scatter of psychiatric illness in the wider family circles of the probands. Nevertheless, apart from frequent marital interactional difficulties there was also a substantial amount of major psychiatric illness and personality disorder among the parents. One father was a severe alcoholic, another had crippling chronic agoraphobia associated with years of psychiatric care in hospital. One mother had killed herself after years of chronic anxiety and depression, a second had a chronic paranoid psychosis, a third had a severe chronic state of anxiety depression. with a variety of phobic avoidance patterns, and a fourth had a history of agoraphobia and depressive illness requiring admission to hospital and electric convulsion therapy. Thus there was evidence that a quarter of the 24 fathers and mothers had or had had formal severe and sometimes chronic psychiatric illness, not infrequently involving social phobic avoidance patterns.

The family conflicts, which we believe to be important contributory factors, took the usual variety of forms (Crisp, 1970) and were in our view linked with the patients' psychopathology, including their abnormal attitudes to food as part of the resolution of their adolescent difficulties.

\section{Patients' History and Illness}

\section{CLINICAL PREVALENCE}

The 11 male patients and two other subjects were seen over a 10-year period during which a series of 160 female patients with primary anorexia nervosa were also referred for diagnosis and treatment. This gives a differential sex prevalence for these 10 years in the clinic of one male to 15 females.

\section{BIRTH WEIGHT AND DURATION OF BREAST-FEEDING}

These data were available on 10 subjects. The mean reported birth weight was $7 \mathrm{lb} 10 \mathrm{oz}$, S.D. $13 \mathrm{oz}(3,450 \mathrm{~g}$, S.D. $370 \mathrm{~g})$. The reported duration of breast-feeding varied from up to three months for eight of the subjects to five and 12 months respectively for the remaining two.

\section{BEHAVIOUR AND NUTRITION IN CHILDHOOD}

This information was available only at the anecdotal descriptive level. As children the probands were reported as having had a variety of neurotic symptoms which neither characterized them as a group nor clearly distinguished them from other children. Several were described as having been "plumpish" but this was not invariable. Most were said to have had good appetites, usually without a history of food fads or other nurturent reflections of mother-child conflict. The parents also tended 
to look back nostalgically on the probands' compliant natures as children.

\section{WEIGHT JUST BEFORE ONSET OF DISORDER}

These weights expressed as a percentage of matched population mean weights are shown in Fig. 1 . It is clear that the probands were an overweight population at this point.

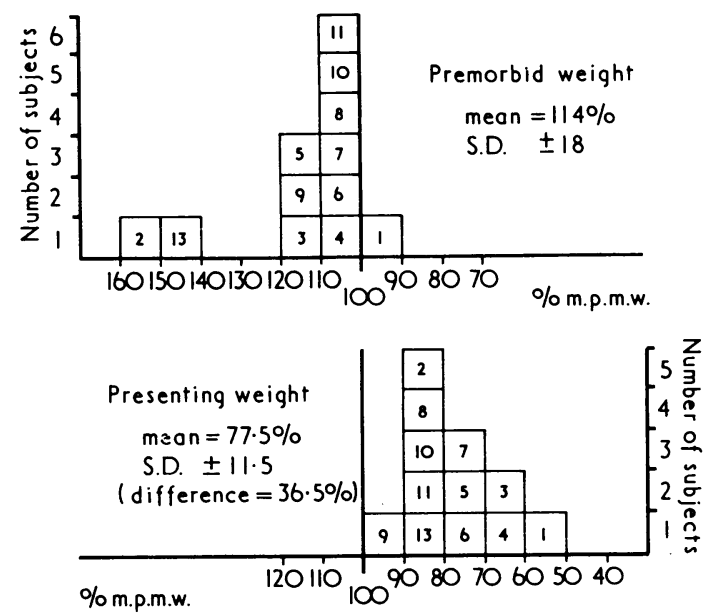

FIG. 1-Premorbid and presenting weights in 12 males. Results given as percentage of matched population mean weight (\% m.p.m.w.) for corresponding ages.

\section{ASPECTS OF CLINICAL STATUS}

The adolescent age of onset of the disorder and the probands' later age of presentation to doctors are shown in Fig. 2. The presenting weight of each subject, again expressed as a percentage of matched population mean weight, is given in Fig. 1.

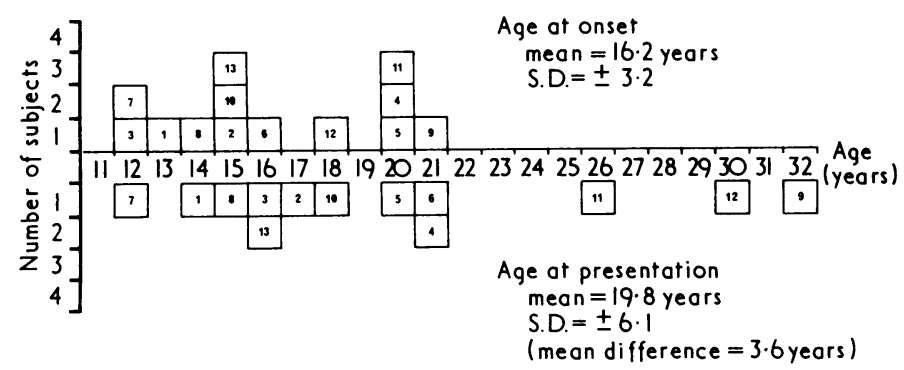

FIG. 2-Reported age of onset and presentation in 13 males with primary anorexia nervosa or weight phobia.

TABLE II-Feeding Habits in the 13 Male Patients

\begin{tabular}{|c|c|c|c|c|c|c|}
\hline \multirow{2}{*}{$\begin{array}{l}\text { Case } \\
\text { No. }\end{array}$} & \multirow{2}{*}{ Age } & \multicolumn{5}{|c|}{ Feeding Activity } \\
\hline & & $\begin{array}{c}\text { Abstain } \\
\text { (A) }\end{array}$ & $\begin{array}{l}\text { Binge- } \\
\text { ing } \\
\text { (B) }\end{array}$ & $\begin{array}{l}\text { Vomit- } \\
\text { ing } \\
\text { (V) }\end{array}$ & $\begin{array}{l}\text { Purging } \\
\text { (P) }\end{array}$ & Alcohol or Drugs \\
\hline \multirow{7}{*}{$\begin{array}{r}1 \\
2 \\
3 \\
4 \\
5 \\
6 \\
7 \\
8 \\
9 \\
10 \\
11\end{array}$} & \multirow{7}{*}{$\begin{array}{l}14 \\
17 \\
16 \\
20 \\
21 \\
22 \\
15 \\
15 \\
34 \\
19 \\
26\end{array}$} & \multirow{7}{*}{$\begin{array}{l}\mathbf{A} \\
\mathbf{A} \\
\mathbf{A} \\
\mathbf{A} \\
\mathbf{A} \\
\mathbf{A} \\
\mathbf{A} \\
\mathbf{A}\end{array}$} & & \multirow{4}{*}{$\begin{array}{l}\mathbf{v}+ \\
\mathbf{v} \dagger \\
\mathbf{v}\end{array}$} & \multirow{3}{*}{$\mathbf{P *}$} & \multirow{8}{*}{$\begin{array}{l}\text { Alcohol } \\
\text { Alcohol } \\
\text { Smoked pot } \\
\text { Alcohol } \\
\text { Occasional } \\
\text { alcohol abuse }\end{array}$} \\
\hline & & & & & & \\
\hline & & & & & & \\
\hline & & & \multirow{5}{*}{$\begin{array}{l}\mathbf{B}^{*} \\
\mathbf{B} \\
\mathbf{B}^{*} \\
\mathbf{B} \\
\mathbf{B}\end{array}$} & & \multirow{5}{*}{$\begin{array}{l}\mathbf{P} * \\
\mathbf{P}\end{array}$} & \\
\hline & & & & \multirow{4}{*}{$V^{*}+$} & & \\
\hline & & & & & & \\
\hline & & & & & & \\
\hline $\begin{array}{l}12 \\
13\end{array}$ & $\begin{array}{l}46 \\
16\end{array}$ & $\begin{array}{l}\mathbf{A} \\
\mathbf{A}\end{array}$ & & & & \\
\hline
\end{tabular}

*Severe.

†Electrolyte disturbance: hypokalaemia.
The feeding patterns are shown in Table II. Patterns of chronic vomiting and purging were predictably associated with severe electrolyte disturbance, most strikingly reflected in severe hypokalemia. Symptomatic epilepsy, based on such metabolic disturbances, was a feature of one and possibly two cases, and was reminiscent of a similar prevalence in the female population (Crisp et al., 1968). Other aspects of behaviour included typical preoccupation with body weight, diets, and cooking; general hyperactivity sometimes incorporated into ritualistic behaviour, nocturnal restlessness, propensities for drug dependence-for example, cigarette smoking and the other drug ingestions noted in Table II-sometimes embarked on in a conscious effort to stave off bulimia and consequent weight gain by stifling appetite and ingesting what was assumed to be a substance of less calorific value, and at other times as a more complex social action; and the absence of sexual activity at either the behavioural or fantasy level.

Apart from the subject in Case 12 another patient (Case 9) had been married. His marriage, established after the onset of the disorder, had been unconsummated and had ended in divorce after five years. The general mood state varied between subjects and was also highly variable in the individual. None showed sustained deep depression or anxiety, but they tended to describe sequential feelings such as panics, elation, anguish, and irritability set against the background of restlessness and, when severely ill, fatigue and exhaustion. At times they would try to assume and convey a manner of blandness, reflecting their acceptance of their present state and their wish not to invoke the concern and attempts of others to change them. They were, however, also able, under certain circumstances, to describe their paralysingly mixed feelings which required them to remain as they were, realizing the hopelessness of their present position but never able to take the fearful step of eating their way to "recovery."

\section{TREATMENT AND OUTCOME}

This report was not written to dwell on individual psychopathology except in so far as its origins are reflected in the relatively "hard" data described above. We believe, however, that we were able to identify overwhelming individual maturational problems in all our patients. These were reflected in the patients' histories as a variety of sexual conflicts and identity crises, often arising within the context of the parental and marital conflicts briefly referred to above.

Subjects tended to be reluctant and sometimes resistant as patients. The 11 who presented as patients had done so only after being "cornered," usually by their general practitioners after attention had been drawn to some superficial but serious aspect of their disorder, such as severe bulimia, vomiting and diarrhoea, symptomatic epilepsy, emaciation, bizarre food fads, or impotence. In these circumstances their complaints took such forms as their desire to eat but inability to do so because of consequent abdominal discomfort or vomiting. We felt justified in effecting the compulsory detention of one patient who was grossly emaciated and weak at presentation but refused to accept treatment.

Six other patients agreed to hospital admission and specific treatment aimed at restoring their weight to their individual matched population mean levels, together with normal dietary intake, as a basis for attempting to help them with the emotional difficulties provoked by such change. One of these cases was reported in detail elsewhere (Crisp and Roberts, 1962). The other six also regained weight precisely in the way described above. Only two patients, however, managed to maintain this and began to explore and cope with their re-emerging maturational problems. Two of the others became bulimic and more alcoholic, as they had feared, and one started regular vomiting for the first time. There was a tendency among two or three of the subjects for antisocial behaviour to re-emerge, which 
had not been evident throughout the duration of the disorder but had sometimes threatened or appeared briefly before in early adolescence.

The case of one patient (Case 7) is reported here in greater detail because the disorder, which started very early in his puberty, clearly stunted his growth, including his stature. It is shown in Fig. 3 that his height remained stationary during the years $12-15$ inclusive. After the initial treatment successfully

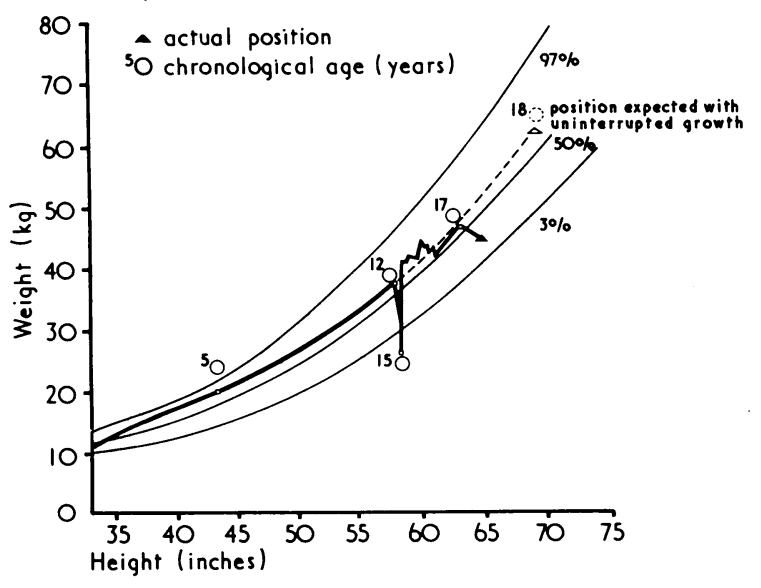

FIG. 3-Case 7. Weight for height with percentiles (Tanner, 1958).

restored his weight to normal he began to grow in height, picking up his growth curve again during the next 18 months. By the end of this period, however, he had lapsed into a mixed state of delinquent behaviour and social phobic avoidance behaviour. He became unmanageable in the general hospital psychiatric unit and was admitted to a psychiatric hospital with the predominant symptom of agoraphobia together with heavy alcohol consumption-the former being the same manifest disorder as his father had experienced since adolescence.

\section{Discussion}

In our view primary anorexia nervosa in the male is probably a rare condition though it may be much more common than is evident in the clinic. In our experience male patients tend to be even more resistant to treatment than females, and even if some features of their disorder are brought to medical attention the fundamental psychopathological abnormalities may remain concealed while the patient allows himself to be investigated for gastrointestinal and endocrine dysfunction or the depressive or obsessional features of his mental state.

The disorder in the male has the same central morbidity, reflecting itself in a phobic avoidance of normal adult weight and attendant impulses and expectations and the consequent social complications and maturational demands. As such it is also a primary disorder of weight and not primarily a feeding disorder. As in the female the adolescent neurotic conflicts which precipitate the disorder appear to be in no way specific to it. In other subjects such conflicts might either be dealt with effectively or promote other disorders of adolescence. We regard the high prevalence of psychiatric illness in the families of these patients, which has been commented on before in relation to female patients with anorexia nervosa (Kay and Leigh, 1954), as another sign of this general underlying neurotic morbidity.

The predisposition to develop primary anorexia nervosa in such circumstances is in our view governed by a variety of factors, among which the major one is dieting behaviour, itself strikingly less common in adolescent males than females.

Such behaviour, often started in an effort to reshape appearance in pursuit of greater attractiveness and self-confidence and a securer identity, may be a response to problems which subsequently lead direct to overdetermination of the dieting behaviour and the development of primary anorexia nervosa. At other times such preliminary dieting appears to have been at first a more independent step. In all instances, however, it appears to provide the initial mechanism of entry into the condition of primary anorexia nervosa, determining the latter's development as the "chosen" defensive posture.

Such behaviour in the cases described occurred against a background of overweight, sometimes though not always severe, and about which the subjects had been sensitive and sometimes teased. Such premorbid overweight often reflects a long-standing state which in females has been found to be associated with faster-than-average growth rate both in the general population and in the population with anorexia nervosa (Crisp, 1970). If this is also true for males it nevertheless remains true that, overall, boys reach puberty some year or so later than girls. Such relative protection from precipitation into adolescence at a very early age may be another factor contributing to the differential sex incidence in the disorder.

It became evident during inquiry and treatment that most of the present male patients had during their early years been overprotected, especially by their mothers. Such concern had often also involved attempts at overnurturing since the time of birth or even conception. These maternal needs, tending to occur in relatively old primiparae, were based on personal or maternal insecurities and anxieties, the ultimate expression of which was sometimes the frank maternal psychiatric disorders referred to above. Not too much can be made of any specific aspects of paternal morbidity, which was more varied in this small series. It was noted, however, that three of the fathers worked with food. Three were contributing acutely to the family instability through their current virtual defections from the marriage. Already as children, then, the patients appeared ill-equipped to enter into and cope with the maturational demands of adolescence and possibly also likely to invoke nurturent means of dealing with such problems. In the three cases in which more than one sibling had had the disorder it appeared to us that they were acting, so to speak, in series, needing in the process to maintain some family interaction and immobilize some threatened change.

The ultimate family basis for the nurturent factors described above probably derives from a complex interaction of genetic and experiential influences. The case of the adoptive family is cited to show the probable important contribution of the latter.

The major effect that the disorder can have on physical growth, especially when its onset is early in adolescence, is shown in Case 7. This finding is in accord with the reported tendency for patients in the parallel female population in whom the disorder has begun during or before the 17th year to be shorter in stature than a population matched for sex, age, and social class. Such retardation and stunting of growth is probably related to malnutrition consequent on predominant carbohydrate starvation but other depletions may be relevant, especially in those subjects given to chronic and often covert vomiting and purging. It is also noteworthy that the above patient complained of severe insomnia; this has been shown elsewhere to be a feature of primary anorexia nervosa, as has a reduction in R.E.M. sleep (Crisp et al., 1971). It has been shown (Sassin et al., 1969) that there is a complex but probably overall direct relation between such forms of sleep and production of growth hormone.

Finally, with this small series it was not thought worth while to compare the course of the disorder in the males with that in the females. Nevertheless, it was our impression that this specific male patient population carried a worse prognosis than the parallel female population.

We would like to acknowledge our gratitude to the large number of other people also concerned in the care of these patients, especially the nursing staff at Atkinson Morley's Hospital and Mrs. B. Harding, psychiatric social worker. 


\section{References}

Bliss, E. L., and Branch, C. H. H. (1960). Anorexia Nervosa. New York, Hoeber.

Bruch, H. (1971). Psychosomatic Medicine, 33, 31

Crisp, A. H. (1967). Hospital Medicine, 1, 713.

Crisp, A. H. (1970). World Review of Nutrition and Dietetics, 12, 452.

Crisp, A. H., Fenton, G. W., and Scotton, L. (1968). British fournal of Psychiatry, 114, 1149.

Crisp, A. H., and Roberts, F. J. (1962). Postgraduate Medical fournal, 38, 350 .
Crisp, A. H., Stonehill, E., and Fenton, G. W. (1971). Postgraduate Medical Fournal, 47, 207.

Dally, P. (1969). Anorexia Nervosa. London, Heinemann.

Kay, D. W. K., and Leigh, D. (1954). Fournal of Mental Science, 100, 411 Sassin, J. F., et al. (1969). Science, 165, 513.

Tanner, J. M. (1958). In Modern Trends in Paediatrics ed. A. Holzel and J. Tizard, p. 331 . London, Butterworths.

Theander, S. (1970). Acta Psychiatrica Scandinavica, Suppl. No. 214.

Toms, D. A., and Crisp, A. H. (1972). Fournal of Psychosomatic Research, 16. In press.

\title{
Effects of Renal Function on Plasma Digoxin Levels in Elderly Ambulant Patients in Domiciliary Practice
}

\author{
E. MARY BAYLIS, M. S. HALL, GILLIAN LEWIS, VINCENT MARKS
}

British Medical fournal, 1972, 1, 338-341

\section{Summary}

An investigation into the relations between the daily dose of digoxin, drug regimen, serum digoxin concentration, and creatinine and digoxin clearance was carried out in a group of elderly ambulant patients in domiciliary practice. Moderate to severe impairment of renal function was found both in patients taking digoxin and in elderly control subjects. Plasma digoxin levels were not related to blood urea concentrations or creatinine clearance. Digoxin clearance was less than creatinine clearance. Now that plasma digoxin levels can be measured relatively easily their estimation should become part of clinical practice.

\section{Introduction}

Despite nearly 200 years of clinical experience since the publication of William Withering's An Account of the Foxglove and Some of its Medical Uses, difficulties are still encountered in prescribing cardiac glycosides. Toxic symptoms are often produced in one patient with doses therapeutically effective for another. With the advent of radioimmunoassay techniques (Smith et al., 1969) for measuring serum digoxin concentrations it has been appreciated that this may be largely due to overlap between toxic and therapeutic blood levels (Chamberlain et al., 1970; Smith and Haber, 1970), although individual variation in sensitivity to the glycosides is important, and other factorsincluding potassium (Sampson et al., 1943), calcium (Moe and Farah, 1965), and magnesium (Seller et al., 1970) concentrations, sodium balance (Harrison and Wakim, 1969), thyroid status (Doherty, 1968), the presence of severe heart disease, or chronic pulmonary disease (Beller et al., 1971)-influence the liability to develop toxicity.

From 67 to $77 \%$ of the digoxin is present in the blood in the protein-free (Evered et al., 1970) pharmacologically active (Lullman and Van Swieten, 1969) state, and its concentration

\footnotetext{
Epsom Hospital Laboratories, West Park Hospital, Epsom, Surrey E. MARY BAYLIS, B.M., B.CH., Senior Registrar in Chemical Pathology GILLIAN LEWIS, B.SC., Biochemist

Forest Row, Sussex

M. S. HALL, M.B., B.S., D.oBST.R.C.o.G., General Practitioner

University of Surrey, Guildford, Surrey

VINCENT MARKS, D.M., F.R.C.P., Professor of Clinical Biochemistry
}

is to a great extent a function of its rate of renal clearance. The tendency for elderly patients to require smaller doses of digoxin to achieve adequate digitalization or therapeutic blood levels (Dall, 1965; Doherty, 1968; Ewy, et al., 1969; Chamberlain et al., 1970) and the poor correlation between serum levels and the daily dose in the presence of impaired renal function (Chamberlain et al., 1970) have been noted, suggesting that renal function should be assessed before prescribing digoxin. In the studies reported (Smith et al., 1969; Chamberlain et al., 1970; Smith and Haber, 1970; Beller et al., 1971) renal function was judged by measuring the blood urea concentration, a notoriously late and insensitive index of renal disease which has moreover been shown on occasion to be well within "normal limits" when the renal digoxin clearance was seriously reduced (Doherty et al., 1967).

More valuable information is provided by measuring the creatinine clearance, and this has been used as a method of adjusting digitalis therapy before blood measurements became available (Jelliffe and Blankenhorn, 1967). Ideally, the serum digoxin concentration should be maintained at a more or less constant, effective level throughout the day, and more accurate knowledge of renal function will influence the dose and mode of administration. Paediatric doses, $0.065 \mathrm{mg}$, may be useful in some cases. This report describes the results of an investigation into the relations between the daily dose of digoxin, drug regimen, serum digoxin concentration, and creatinine clearance in a group of elderly ambulant patients in general practice.

\section{Patients and Methods}

Thirty-one randomly selected subjects who had clinically been well controlled by a constant dose of digoxin for at least one month were investigated. Blood samples were taken when these patients presented at the surgery, either spontaneously (usually to obtain a repeat digoxin prescription) or by request. Clinical details recorded in each case include: name, age, date of birth, sex; nature of the heart condition; other significant diseases present; drugs; digoxin therapy (with details of dosage, duration of therapy, frequency of administration, and exact time relations between venepuncture and taking the previous dose); and history of symptoms which could be due to digoxin therapy.

Second and sometimes third blood samples were collected from most patients at a prearranged time of day to gain further information about the plasma digoxin level at varying intervals after taking the drug. In 25 patients creatinine clearances were measured. Each patient was given precise verbal and written instructions how to collect a 24-hour sample of urine and was visited during that period for blood sampling. (Only one blood 